\title{
COVID-19 no período gestacional: uma revisão integrativa da literatura
}

\author{
COVID-19 in the gestational period: an integrative literature review \\ COVID-19 en el período gestacional: una revisión integradora de la literature
}

Recebido: 15/09/2021 | Revisado: 20/09/2021 | Aceito: 22/09/2021 | Publicado: 24/09/2021

\author{
Luana Aparecida Correa Dezincourt \\ ORCID: https://orcid.org/0000-0002-7816-1926 \\ Centro Universitário São Lucas, Brasil \\ E-mail: dezincourtluana@gmail.com \\ Antonio Rodrigues da Silva Neto \\ ORCID: https://orcid.org/0000-0003-0054-510X \\ Universidade Estadual do Piauí, Brasil \\ E-mail: antonionettors5@gmail.com \\ Dakson Douglas Araújo \\ ORCID: https://orcid.org/0000-0003-0102-7240 \\ Universidade Federal do Delta do Parnaíba, Brasil \\ E-mail: dacksondouglas@gmail.com \\ Aline Munarini de Quevedo Carvalho \\ ORCID: https://orcid.org/0000-0002-6864-4561 \\ Centro Universitário São Lucas, Brasil \\ E-mail: alinemq.carvalho@hotmail.com \\ Sthefanie da Silva Bessa \\ ORCID: https://orcid.org/0000-0001-7562-1643 \\ Centro Universitário São Lucas, Brasil \\ E-mail: sthefaniesbessa@gmail.com \\ Jhersyka Kessin Gonçalves Carvalho Campos \\ ORCID: https://orcid.org/0000-0002-7106-594X \\ Centro Universitário São Lucas, Brasil \\ E-mail: jhersyka_k@hotmail.com \\ João Pedro Ribeiro Santiago Ferreira \\ ORCID: https://orcid.org/0000-0002-9371-359X \\ Centro Universitário São Lucas, Brasil \\ E-mail: Joopedro_f@hotmail.com \\ Amanda Ferreira Negri \\ ORCID: https://orcid.org/0000-0003-1442-4069 \\ Centro Universitário São Lucas, Brasil \\ E-mail: amandafnegri@hotmail.com \\ Gabriella Linhares de Andrade \\ ORCID: https://orcid.org/0000-0003-2481-9646 \\ Universidade Federal do Delta do Parnaíba, Brasil \\ E-mail: gabriellalinhares9@gmail.com \\ Kerlane Alves Fernandes \\ ORCID: https://orcid.org/0000-0002-4078-2727 \\ Universidade Estadual do Piauí, Brasil \\ E-mail: kerlanealves@gmail.com \\ Gabriel e Silva Sales \\ ORCID: https://orcid.org/0000-0003-0769-3447 \\ Universidade Federal do Piauí, Brasil \\ E-mail: gabriel.sales20093@gmail.com \\ Ana Karina Borges Costa \\ ORCID: https://orcid.org/0000-0001-9006-6545 \\ Universidade Estadual do Piauí, Brasil \\ E-mail: a.karinaborges@gmail.com
}

\section{Resumo}

O novo coronavírus, SARS-CoV-2, responsável pela infecção da COVID-19, teve seu primeiro caso na cidade de Whuan localizado na China, desde então, tem se propagado pelo mundo inteiro tornando-se um grave problema de saúde pública. Com uma alta taxa de transmissão, foi considerado que alguns grupos de pessoas eram mais prédispostos a desenvolver a forma grave da doença, dentre estes, as gestantes. Nesse contexto, vale atribuir que o período gravídico é marcado por inúmeras mudanças fisiológicas, psicológicas e pessoais, que exigem maior atenção à saúde da mulher. A assistência ao pré-natal é determinante para o bem-estar da mãe e do bebê, além de diminuir potencialmente as principais causas de mortalidade materna e neonatal. Diante disso, o presente estudo objetivou-se averiguar, através de uma revisão bibliográfica integrativa, quais as principais implicações atreladas à infecção pelo Sars-Cov-2 durante o período gestacional e como acontece este atendimento e auxílio das grávidas. Nesta pesquisa 
foram utilizadas as bancas de dados nas plataformas digitais ScienceDirect e Pubmed. As buscas dos artigos consistiu entre o período de 2020 e 2021. Verificou-se que a falta de conhecimento e incertezas do SARS-CoV-2 e principalmente os impactos que a infecção viral poderiam gerar nas gestantes propiciou para que medidas conformacionais fossem tomadas a fim de protegê-las. Portanto, a implementação de políticas públicas substituíram as visitas domiciliares e o volume de consultas hospitalares, e adequariam a telemedicina para não as deixar desassistidas. Portanto, busca-se mais estudos que possam fundamentar e alicerçar as ideias sobre essa temática que ainda é preocupante.

Palavras-chave: COVID-19; Gravidez; Complicações na gravidez.

\begin{abstract}
The new coronavirus, SARS-CoV-2, responsible for the COVID-19 infection, had its first case in the city of Whuan located in China, since then it has spread all over the world, becoming a serious public health problem. With a high transmission rate, it was considered that some groups of people were more predisposed to develop the severe form of the disease, among them, pregnant women. In this context, it is worth noting that the pregnancy period is marked by numerous physiological, psychological and personal changes, which require greater attention to women's health. Prenatal care is crucial for the well-being of the mother and baby, in addition to potentially reducing the main causes of maternal and neonatal mortality. Therefore, the present study aimed to investigate, through an integrative literature review, which are the main implications linked to the infection by Sars-Cov-2 during the gestational period and how this care and assistance to pregnant women takes place. In this research, databases in the digital platforms ScienceDirect and Pubmed were used. The searches for articles consisted between the period 2020 and 2021. It was found that the lack of knowledge and uncertainties of SARS-CoV-2 and especially the impacts that viral infection could generate in pregnant women allowed for conformational measures to be taken in order to protect them. Therefore, the implementation of public policies replaced home visits and the volume of hospital appointments, and would adapt telemedicine so as not to leave them unattended. Therefore, more studies are sought that can substantiate and support ideas on this topic that is still worrisome.
\end{abstract}

Keywords: COVID-19; Pregnancy; Pregnancy complications.

\begin{abstract}
Resumen
El nuevo coronavirus, SARS-CoV-2, responsable de la infección COVID-19, tuvo su primer caso en la ciudad de Whuan ubicada en China, desde entonces se ha extendido por todo el mundo, convirtiéndose en un grave problema de salud pública. Con una alta tasa de transmisión, se consideró que algunos grupos de personas estaban más predispuestos a desarrollar la forma severa de la enfermedad, entre ellos, las mujeres embarazadas. En este contexto, cabe señalar que el período de embarazo está marcado por numerosos cambios fisiológicos, psicológicos y personales, que requieren una mayor atención a la salud de la mujer. La atención prenatal es fundamental para el bienestar de la madre y el bebé, además de reducir potencialmente las principales causas de mortalidad materna y neonatal. Por tanto, el presente estudio tuvo como objetivo investigar, a través de una revisión integradora de la literatura, cuáles son las principales implicaciones vinculadas a la infección por Sars-Cov-2 durante el período gestacional y cómo se lleva a cabo este cuidado y asistencia a la gestante. En esta investigación se utilizaron bases de datos en las plataformas digitales ScienceDirect y Pubmed. Las búsquedas de artículos se realizaron entre el período 2020 y 2021 . Se encontró que el desconocimiento e incertidumbre del SARS-CoV-2 y especialmente los impactos que la infección viral podría generar en mujeres embarazadas permitieron tomar medidas conformacionales para Protegelos. Por tanto, la implementación de políticas públicas reemplazó las visitas domiciliarias y el volumen de consultas hospitalarias, y adecuaría la telemedicina para no dejarlas desatendidas. Por ello, se buscan más estudios que puedan sustentar y sustentar ideas sobre este tema que aún es preocupante.
\end{abstract}

Palabras clave: COVID-19; El embarazo; Complicaciones del embarazo.

\title{
1. Introdução
}

O novo coronavírus, Sars-Cov-2, agente etiológico responsável pela infecção da COVID-19, teve seu primeiro caso na cidade de Whuan - China e, desde então, tem se propagado pelo mundo inteiro, vulnerabilizando os mais variados grupos (Estrela et al., 2020). Sua transmissão ocorre através do contato com secreções respiratórias dos infectados em superfícies contaminadas ou gotículas e aerossóis expelidos por indivíduos acometidos pela doença (Johansson et al., 2021; Sousa et al., 2020).

O período de incubação dura em média cinco dias, evoluindo de forma assintomática ou sintomática. Evidências sugerem que a maioria das transmissões ocorrem de pessoas sintomáticas para outras, contudo, muitos pacientes podem transmitir ainda no período de incubação. É comum a manifestação de sintomas como febre, tosse, congestão nasal, fadiga e outros sinais de infecção de via aérea superior com possibilidade de agravamento do quadro infeccioso, progredindo com 
acometimento pulmonar, desconforto respiratório, diminuição da saturação de oxigênio e distúrbios hemodinâmicos que levam ao aumento significativo da mortalidade (Velavan \& Meyer, 2020).

Nesse contexto, a Organização Mundial da Saúde (OMS) adotou medidas de segurança com o objetivo de reduzir a transmissibilidade do vírus, como distanciamento social, higienização das mãos, uso de máscaras, limpeza de ambientes, isolamento de casos suspeitos, confirmados e seus contatos, principalmente àqueles classificados como grupo de risco para o agravamento da COVID-19 portadores de doenças crônicas, idosos, gestantes, puérperas, crianças menores que cinco anos e populações vulneráveis (Silva et al., 2020).

Hospitais de campanha foram construídos para atender a demanda de uma pandemia com a urgência necessária e o isolamento social tornou-se o principal meio de evitar sua disseminação, sendo indispensável para o melhor controle (Ruprecht, 2020). Contudo, a demanda de profissionais e recursos desviou consideravelmente a atenção de outros grupos que carecem de serviços de saúde independente das circunstâncias, dentre eles, as gestantes.

O período gravídico é marcado por inúmeras mudanças fisiológicas, psicológicas e pessoais que exigem maior atenção à saúde da mulher, e a assistência pré-natal é determinante para o bem-estar da mãe e do bebê, além de diminuir potencialmente as principais causas de mortalidade materna e neonatal (Brasil, 2012). Dessa forma, é necessário considerar o impacto da pandemia e isolamento social na vida da gestante e garantir um acompanhamento adequado que é indispensável ao período, sendo este limitado pela quantidade e qualidade de suas consultas (Núbia, 2020; Da Costa et al., 2020).

As características clínicas em mulheres grávidas ainda são desconhecidas, bem como a possibilidade de transmissão vertical de pneumonia por COVID-19. A imunossupressão fisiológica do período gestacional confere ao organismo materno uma maior susceptibilidade a infecções virais (Souza et al., 2020) e desenvolvimento de formas graves de doenças caso venha a ser infectado. Embora o risco da gestação em um período pandêmico de SARS-CoV-2 seja facilmente explicado, poucas são as evidências sobre as reais complicações dessa nova variante para a gestante ou para o feto.

Outras alterações fisiológicas da gravidez, sobretudo nos sistemas respiratório e circulatório, podem piorar o quadro clínico das mulheres infectadas por um vírus durante a gestação (Ramussem et al., 2020) e, se tratando de uma infecção por SARS-CoV-2, evoluir para a síndrome respiratória aguda grave. Mulheres grávidas, são menos propensas a apresentar sintomas como febre, dispneia e mialgia. Entretanto, acabam tendo maior chance de ter um parto prematuro, com um risco elevado de morte materna e uma grande probabilidade de serem admitidas em UTIs, precisando utilizar ventilação invasiva (Allotey et al., 2020).

Evidências apontam que as novas variantes têm ação mais agressiva em gestantes e, por conta disso, postergar os planos de gravidez têm sido recomendados, segundo o secretário de Atenção Primária à Saúde (SAPS) do Ministério da Saúde, Raphael Câmara (Cardim, 2021). Em meio a tantas incertezas, é compreensível o sentimento de angústia vivenciado pelas gestantes em período de pandemia e essa realidade confere transtornos emocionais que reverberam em uma experiência negativa de gravidez, gerando impactos ainda mais significativos de ordem psicológicas.

Portanto, o presente estudo visa averiguar, através de uma revisão bibliográfica integrativa, quais as principais implicações atreladas à infecção pelo SARS-CoV-2 durante o período gestacional e como acontece este atendimento e auxílio das grávidas, a fim de se fazer indispensável que os profissionais de saúde estejam atualizados e treinados para a tomada de decisões diante esse cenário desafiador.

\section{Metodologia}

O estudo trata-se de uma revisão bibliográfica integrativa, de natureza descritiva e com abordagem qualitativa. Esta revisão é alicerçada seguindo a construção de seis etapas: escolha do tema e o levantamento de uma problemática para a pesquisa, coleta de dados e definição dos critérios de inclusão e exclusão, análise de estudos pré-selecionados, descrição de 
estudos selecionados, análise e interpretação dos resultados, apresentação e resumo da ideia. A pesquisa preza por fundamentar e colaborar com a temática de auxilio e atendimento prestados à gravidas em tempos de pandemia de COVID-19 (Ferreira et al., 2021).

A pesquisa bibliográfica tem finalidade de reunir diversas informações que possa vir a fomentar os estudos já existentes nesta área do conhecimento que servirá de base para a construção deste tema para isso foram utilizadas bancos de dados: ScienceDirect e Pubmed. A busca foi realizada a partir da utilização de descritores que trabalham a temática da pesquisa de forma constatada pelo vocabulário estruturado DeCS - Descritores em Ciência da Saúde. Sendo utilizado os termos "Grávidas", “Atendimento", "Período Gestacional” e "Covid-19” para a busca nos títulos, abstract ou corpo do estudo. Pesquisou-se artigos originais sobre o tema, publicados entre 2020 e maio de 2021.

A análise destes artigos e seus dados foram definidas pela leitura e verificação a partir dos resumos de todos os resultados encontrados, para pré-definir quais seriam incluídos e excluídos da pesquisa. Nesse sentido, definiu-se que seriam incluídas produções disponíveis na íntegra, nos idiomas português, inglês e espanhol, que permitam uma melhor coerência com a temática desenvolvida nesta pesquisa. Foram excluídas publicações que não problematizavam sobre a assistência e atendimento a gravidas em tempos de pandemia da COVID-19. O fluxograma, logo abaixo demonstra o processo de seleção dos artigos.

Fluxograma 1 - Fluxograma dos resultados de busca das publicações segundo os objetivos do presente estudo.

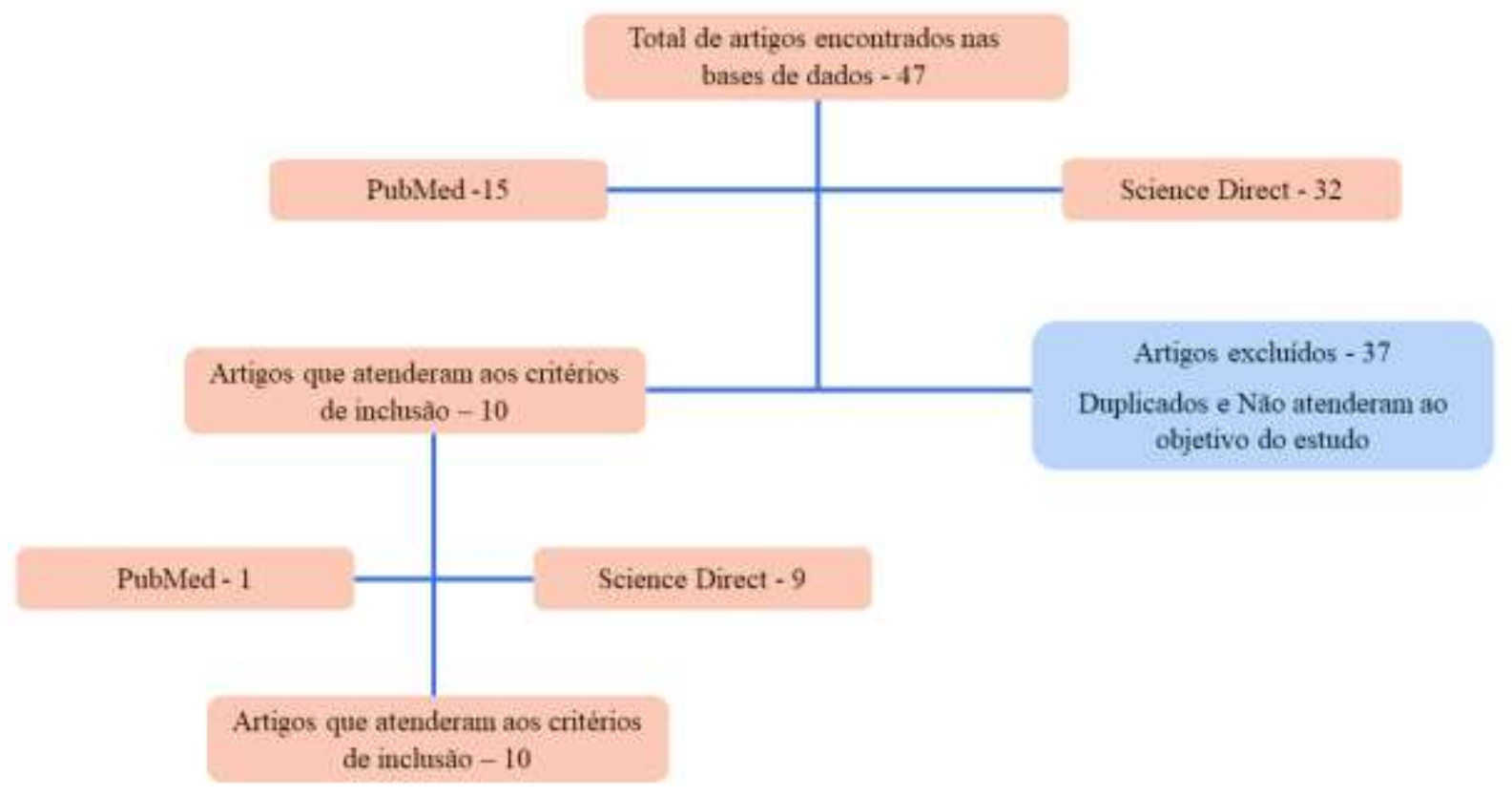

Fonte: Autores (2021).

\section{Resultados e Discussão}

Para a busca de dados, foram utilizadas duas palataformas, respectivamente, ScienceDirect e Pubmed. Ao todo foram selecionados 47 artigos para essa revisão integrativa, 15 encontrados na plataforma Pubmed e 32 encontrados na plataforma Science Direct. Obedecendo os critérios de inclusão e exclusão, restaram 10 artigos, onde foram utilizados para essa revisão integrativa, sendo 1 artigo da plataforma PubMed e 9 artigos da plataforma Science Direct, todos dispostos em língua inglesa. 
Quadro 1 - Descrição dos estudos que atenderam aos critérios de elegibilidade segundo autor e ano de publicação, título e objetivo do estudo.

\begin{tabular}{|c|c|c|c|}
\hline Autores/Ano & Título & Objetivo de Estudo & Banco de dados \\
\hline $\begin{array}{l}\text { Meaney et al., } \\
2020\end{array}$ & $\begin{array}{l}\text { The impact of COVID- } 19 \text { on pregnant } \\
\text { womens' experiences and perceptions of } \\
\text { antenatal maternity care, social support, } \\
\text { and stress-reduction strategies }\end{array}$ & $\begin{array}{c}\text { Avaliar a satisfação das mulheres grávidas com os } \\
\text { cuidados pré-natais e apoio social e examinar as } \\
\text { estratégias de redução do estresse usadas pelas } \\
\text { mulheres durante a pandemia. }\end{array}$ & ScienceDirect \\
\hline $\begin{array}{l}\text { Vasileyski et al., } \\
2021\end{array}$ & $\begin{array}{l}\text { Receiving maternity care during the } \\
\text { COVID-19 pandemic: Experiences of } \\
\text { women's partners and support persons }\end{array}$ & $\begin{array}{l}\text { Explorar as experiências de parceiros e pessoas de } \\
\text { apoio de mulheres que receberam cuidados de } \\
\text { maternidade durante a pandemia COVID-19. }\end{array}$ & ScienceDirect \\
\hline $\begin{array}{l}\text { Sheil \& Mcauliffe } \\
2021\end{array}$ & $\begin{array}{l}\text { Reorganisation of obstetric services } \\
\text { during the COVID pandemic e } \\
\text { Experience from National Maternity } \\
\text { Hospital Dublin Ireland }\end{array}$ & $\begin{array}{c}\text { Descrever a jornada do National Maternity } \\
\text { Hospital Dublin em reorganizar os serviços para } \\
\text { permitir a continuidade dos cuidados às gestantes. }\end{array}$ & ScienceDirect \\
\hline $\begin{array}{l}\text { Chmielewska et } \\
\text { al., } 2021\end{array}$ & $\begin{array}{l}\text { Effects of the COVID- } 19 \text { pandemic on } \\
\text { maternal and perinatal outcomes: a } \\
\text { systematic review and meta-analysis }\end{array}$ & $\begin{array}{c}\text { Avaliar a evidência coletiva sobre os efeitos da } \\
\text { pandemia nos resultados maternos, fetais e } \\
\text { neonatais. }\end{array}$ & ScienceDirect \\
\hline Ashish et al., 2020 & $\begin{array}{l}\text { Effect of the COVID-19 pandemic } \\
\text { response on intrapartum care, stillbirth, } \\
\text { and neonatal mortality outcomes in } \\
\text { Nepal: a prospective observational study }\end{array}$ & $\begin{array}{l}\text { Avaliar o número de partos institucionais, seus } \\
\text { resultados (natimortos institucionais e taxa de } \\
\text { mortalidade neonatal) e a qualidade do } \\
\text { atendimento durante o parto antes e durante o } \\
\text { bloqueio nacional COVID-19 no Nepal. }\end{array}$ & ScienceDirect \\
\hline $\begin{array}{l}\text { Bradfield et al., } \\
\quad 2021\end{array}$ & $\begin{array}{l}\text { Midwives' experiences of providing } \\
\text { maternity care during the COVID-19 } \\
\text { pandemic in Australia }\end{array}$ & $\begin{array}{c}\text { Explorar e descrever as experiências das parteiras } \\
\text { na prestação de cuidados de maternidade durante a } \\
\text { pandemia COVID-19 na Austrália. }\end{array}$ & ScienceDirect \\
\hline $\begin{array}{l}\text { Vazquez et al., } \\
2020\end{array}$ & $\begin{array}{l}\text { The impact of the Covid-19 lockdown } \\
\text { on the experiences and feeding practices } \\
\text { of new mothers in the UK: Preliminary } \\
\text { data from the COVID-19 New Mum } \\
\text { Study }\end{array}$ & $\begin{array}{l}\text { Descrever e comparar as experiências de parto e } \\
\text { pós-natal de mulheres que deram à luz antes (BL) } \\
\text { e durante (DL) o bloqueio. }\end{array}$ & ScienceDirect \\
\hline Verma et al., 2020 & $\begin{array}{l}\text { Neonatal intensive care unit } \\
\text { preparedness for the Novel Coronavirus } \\
\text { Disease-2019 pandemic: A New York } \\
\text { City hospital perspective }\end{array}$ & $\begin{array}{l}\text { Fornecer orientações básicas consolidadas e listas } \\
\text { de verificação para os médicos nas unidades de } \\
\text { terapia intensiva neonatal nos principais aspectos } \\
\text { da preparação necessária para combater a } \\
\text { exposição ou infecção com COVID-19 }\end{array}$ & ScienceDirect \\
\hline $\begin{array}{l}\text { Peahl \& Howell } \\
\quad 2020\end{array}$ & $\begin{array}{c}\text { The Evolution of Prenatal Care Delivery } \\
\text { Guidelines in the United States }\end{array}$ & $\begin{array}{l}\text { Obter uma visão melhor ao compreender a } \\
\text { evolução das diretrizes de assistência pré-natal em } \\
\text { tempos de pandemia. }\end{array}$ & ScienceDirect \\
\hline $\begin{array}{l}\text { Quansar et al., } \\
2020\end{array}$ & $\begin{array}{l}\text { Attitude and practices related to } \\
\text { coronavirus disease (COVID-19) } \\
\text { pandemic among pregnant women } \\
\text { attending family welfare clinic amid } \\
\text { Phase-2 lock down }\end{array}$ & $\begin{array}{l}\text { Conhecer a percepção e a prática dos pacientes de } \\
\text { check-ups de ANC (ANCs) em relação ao } \\
\text { COVID-19 e quais as implicações disso em seus } \\
\text { check-ups de rotina. }\end{array}$ & PubMed \\
\hline
\end{tabular}

Fonte: Autores (2021). 


\subsection{Atenção à saúde das gestantes com diagnóstico de infecção pelo SARS-CoV-2}

As vulnerabilidades fisiológicas enfrentadas pelas gestantes, principalmente diante a possibilidade de infecção pelo SARS-CoV-2, trouxeram à tona preocupações quanto ao retrocesso de décadas de investimento na redução da mortalidade materna e infantil em locais de poucos recursos, como evidenciado por Chmielewska et al. (2020). Embora seu estudo tenha mostrado o aumento da natimortalidade e da taxa de mortalidade materna consonante à pandemia, não se sabe se estas foram consequências diretas da infecção pelo vírus ou quais foram os mecanismos dessas mudanças. Uma explicação proposta para o aumento desses resultados adversos da gravidez seria a redução do acesso aos cuidados às gestantes. Nesse contexto, Vasileyske (2021) propõe, em seu trabalho, que os serviços de assistência à maternidade busquem estratégias alternativas para atender às gestantes.

Para contornar essa necessidade, Sheil e McAuliffe (2021) elucidam maneiras pelas quais os serviços obstétricos foram reorganizados de forma a continuar a cuidar das gestantes durante o cenário da pandemia de COVID-19, incluindo mudanças na trama de cuidados específicos necessários às gestantes infectadas pelo vírus dentro do National Maternity Hospital (NMH), na Irlanda. Através de melhorias nos serviços e fluxo de pacientes, adaptações de infraestrutura, higiene e políticas de visitantes, desenvolvimento de uma abordagem física concomitante à consulta virtual para promoção de educação em saúde foi possível proteger tanto as pacientes quanto a equipe de saúde e conduzir de forma bem sucedida a gravidez e o nascimento, independentemente de uma pandemia.

No mesmo estudo, foi evidenciado o desenvolvimento da abordagem de "hospital dentro de um hospital", facilitada pelo adiamento das clínicas ginecológicas e da cirurgia ginecológica eletiva, usufruindo da equipe e do espaço para o desenvolvimento de vias de fluxo diferentes para pacientes suspeitas ou confirmadas de SARS-CoV-2, permitindo que essas pacientes fossem atendidas em ambiente apropriado, com equipe protegida e tratadas separadamente, ao mesmo tempo que garantiu toda a gama de suporte clínico necessário às gestantes infectadas.

\subsection{Atendimento e acolhimento a gestantes em tempos de pandemia da COVID-19}

O estudo realizado por Quansar et al. (2020) sobre a percepção das pacientes atendidas em pré-natal quanto à atitude e práticas relacionadas ao COVID-19 demonstrou que dentre a maioria que participou tinha idade entre 26 e 35 anos, moradoras de zona urbana com ensino médio ou superior. A preocupação acerca da letalidade que a infecção do novo coronavírus poderia causar entre as gestantes era comum, bem como o medo de que alguém assintomático e que morasse por perto da família pudesse transmiti-las o novo vírus e colocá-las em risco. Apesar de o estudo não mostrar como eram realizados os atendimentos no período de pré-natal nos ambulatórios, percebe-se que as escolhas e necessidades quanto ao local de parto, atenção à saúde da gestante entre outras dúvidas corriqueiras desse período eram bem solucionadas pelos funcionários dos ambulatórios.

A pandemia da COVID-19 sobrepujou as necessidades básicas do atendimento médico, não só da ginecologia e obstetrícia, mas de várias outras especialidades, o que tornou comum as ausências de atendimentos mais aprimorados a esse grupo. No estudo de Meaney et al. (2020) ainda que a satisfação com o atendimento pré-natal de acordo com o número de consultas tenha sido considerado adequado por mais da metade das entrevistadas, algumas mulheres relataram que o tempo entre as consultas era muito longo e outras consideraram-se insatisfeitas. O que coloca em risco a segurança da gestante e do bebê e isso é corroborado pela situação que a pandemia da COVID-19 imprime.

Por outro lado, as participantes relataram que a gravidez ficou mais estressante pelo impacto da pandemia em diferentes cenários de suas vidas, principalmente, pela insatisfação com as mudanças nos serviços de maternidade implantados, como preparação pré-natal, aulas de preparação parental e consultas canceladas ou adiadas. $\mathrm{O}$ apoio social, sobretudo por seus 
entes, alterou significativamente suas percepções devido às restrições com da maternidade, o que também se tornou fator de estresse (Meaney et al., 2020).

Enquanto que o estudo de Vasileyski et al. (2021) demonstrou em seus resultados que parceiros e outros familiares das mulheres grávidas também tiveram uma sensação de perda em relação a atenção e cuidado com a gravidez na pandemia da COVID-19 devidos às limitações, além de relatarem sentimentos de isolamento e sofrimento psicológico alguns conflitos e incertezas de informações dentro dos serviços de maternidade, o que se mostra semelhante aos resultados encontrados por Meaney et al. (2020), uma vez que em ambos os estudos os grupos mostraram-se insatisfeitos com o impacto negativo que a pandemia propiciou não só às gestantes, mas sobretudo nas relações familiares.

No estudo por sua vez de Sheil e Mcauliffe (2021) realizado na Irlanda mostrou que a reorganização intra-hospitalar de fluxos e condutas seriam peças chave no momento de pandemia, tendo em vista a necessidade que a situação mundial em diferentes setores, e na saúde não seria diferente, de readaptação de modelos organizacionais para atender a nova realidade. Os serviços foram alterados conforme orientações nacionais e internacionais, dentre esses, cirurgias eletivas e colposcopia ambulatoriais inicialmente foram canceladas a partir do início de março. Os ambulatórios de maternidade foram priorizados, com pacientes telefonando antes da consulta planejada.

Além disso, parte da anamnese, como história obstétrica era realizada por telefone a fim de otimizar o atendimento em meio à pandemia, além de consultas presenciais e virtuais. Os serviços de obstetrícia foram aumentados com mais visitas domiciliares e instalações de teste COVID-19 para pacientes e funcionários foram intensificadas para garantia da atenção à saúde das gestantes no período (Sheil \& Mcauliffe, 2021).

Esse estudo revelou-se diferente dos resultados mostrados por Meaney et al. (2020) e Vasileyski et al. (2021), pois para que conseguissem atender a população de gestantes, bem como acompanhá-las de forma mais cuidadosa durante a gestação em período de pandemia. Outro fato foi que os diferentes meios adotados foram fundamentais para a integralidade do cuidado.

Vazquez et al. (2020) revela que outro motivo de sofrimento psicológico para a gestante era o fato de que os familiares não pudessem ver o bebê após o nascimento em detrimento do distanciamento social, o que gerava sentimentos angústias e desespero. Deve-se considerar que na maioria dos estabelecimentos de saúde se encontravam despreparados, e isso corroborou para muitas questões, sobretudo a psicológica.

O que foi diferente já no serviço irlandês mostrado por Sheil e Mcauliffe (2021), em que a reorganização de atendimento para o cenário de pandemia foi consolidado para um atendimento satisfatório. Essa diferença pode ser atribuída ao fato que cada país, assim como estado e cidade, tem liberdade de gerenciar suas atividades de acordo com a necessidade, com isso pode ser a razão desta diferença em relação aos atendimentos.

A pesquisas voltadas ao atendimento e acolhimento com mulheres foram conduzidos em diversos locais do mundo em busca de observar as mudanças ocorridas devido à situação pandêmica. Neste sentido, a maioria dos trabalhos relatam que as mulheres, independente dos locais onde estão descrevem que o suporte médico tanto na grávida como nas puérperas foi negativamente modificado, uma vez que, a assistência à saúde se tornou insuficiente, e a procura por profissionais de apoio para a saúde mental foi uma alternativa para driblar as crises da fase na pandemia da COVID-19.

\subsection{Segurança às grávidas em tempos de pandemia COVID-19}

Durante a pandemia, a diminuição dos serviços oferecidos às gestantes proporcionou impactos negativos à experiência gestacional tanto quanto a vulnerabilidade causada pela falta de conhecimento acerca dos desdobramentos da infecção por SARS-CoV-2 no organismo materno. Buscando prover a segurança necessária a esse grupo, mudanças nas diretrizes de atendimento pré-natal foram necessárias. Segundo Peahl e Howell (2020), medidas como adoção de visitas 
virtuais e redução dos horários de visitas, ainda que forçadas pelo contexto, foram evoluções necessárias para moldar o futuro do cuidado pré-natal, sem regredir ao tipo de assistência desestruturada de décadas atrás e suas altas taxas de mortalidade materna e infantil, como contextualizado pelos autores.

No estudo de Sheil e McAuliffe (2021), foram incorporadas medidas para redução das consultas presenciais, como aumento de visitas domiciliares, inclusão de consultas virtuais e de "visitas" pós parto por telefone, e substituição dos serviços programados de educação individual e em grupo por sessões virtuais com desenvolvimento de uma plataforma de educação digital onde foram entregues componentes de educação pré-natal, intraparto e pós-natal possibilitando uma gestação tranquila sob qualquer circunstância. Os autores manifestam uma abordagem já visualizada por Peahl e Howell (2020) - a importância de considerar mudanças no atendimento pré-natal como forma de garantir segurança às pacientes e, consequentemente, aprender, diante de um momento desafiador e incerto, a adaptar a assistência às gestantes.

Por outro lado, essas restrições implementadas nos serviços de maternidade são entendidas por Meaney et al. (2021) como limitantes de interações entre as pacientes e os profissionais de saúde responsáveis por assisti-las, culminando no sentimento de insegurança, por parte das gestantes em relação aos cuidados e apoio durante a gravidez, com consequências a curto e longo prazo para a saúde das mulheres e das crianças. Embora estivessem cientes da situação de vulnerabilidade, pouco foram informadas acerca dos reais riscos aos quais estão expostas, padecendo em sentimento de abandono e angústias (Vasilevski et al., 2021).

Dessa forma, é nítida a necessidade de maior suporte psicossocial à gestante durante a pandemia, principalmente diante desse cenário de difusão da telemedicina e emprego de novas abordagens de consultas virtuais e telefônicas. Nesse contexto, é imprescindível o fornecimento e fácil acesso a todo tipo de informação, para que não haja a diminuição da qualidade dos cuidados pré-natais, percepção de desamparo e desproporcionalidade ao contexto hospitalar, quando comparadas às consultas presenciais. Além disso, é indispensável que toda equipe multidisciplinar responsável pelo acolhimento desse grupo seja adequada a novos padrões de proteção ao atendimento, como consultas previamente marcadas a evitar aglomerações e triagens com aplicação de teste rápido para a detecção de SARS-CoV-2, proporcionando uma abordagem clínica abrangente e integralizada, além de proteção a todos os envolvidos, especialmente às gestantes.

\section{Conclusão}

Inegavelmente a pandemia causada pelo SARS-CoV-2 atingiu negativamente todos os âmbitos, que vai desde do setor educação, comércio, segurança pública e saúde, incluindo o período gestacional de muitas mulheres. O atendimento e acolhimento a gestantes nesse período sofreu várias alterações, com os hospitais lotados e priorizando não expor as gestantes ao risco de contrair o vírus, muitas consultas e até mesmo aulas de preparação parental foram adiadas. Esse cenário de incertezas, medo e estresse corroborou para o desenvolvimento de um quadro de sofrimento psicológico.

O conhecimento com um material reduzido e cercado de incertezas do SARS-CoV-2 e principalmente os impactos que a infecção viral poderiam gerar nas gestantes propiciou para que medidas conformacionais fossem tomadas a fim de protegê-las. Políticas públicas substituíram as visitas domiciliares e o volume de consultas hospitalares, e adequaram a telemedicina para não as deixar desassistidas, além do âmbito hospitalar onde o horário de visitas familiares foi reduzido. Um contexto que priorizou a saúde física da gestante, mas que deixou a saúde mental vulnerável.

A atenção à saúde das gestantes diagnosticadas com a infecção pelo SARS-CoV-2 foi outro motivo que propiciou para adaptações de infraestrutura, higiene e políticas de visitantes. A imunossupressão, apesar de ser fisiológica no período gestacional, as torna ainda mais susceptíveis à possíveis complicações causadas pelo fator viral, um cenário que poderia levar a um grande retrocesso na medicina: a luta contra a mortalidade materna, bem como a manutenção da saúde psíquica em meio às questões de saúde pública como o isolamento social e vacinação em massa. 
Dessa forma, observa-se uma necessidade crescente com a gestante no período pandêmico. Um cuidado integral que priorize a saúde física e mental, visto que a maioria dos artigos selecionados pontuaram negativamente para as gestantes no período pandêmico. É imprescindível continuar aprimorando a forma de assistir as gestantes não só em ambiente hospitalar, mas também na rede de acolhimento, visando amenizar impactos psicológicos como o medo, ansiedade e as incertezas relacionadas a gestação em período pandêmico.

\section{Referências}

Afshar, Y., Gaw, S. L., Flaherman, V. J., Chambers, B. D., Krakow, D., Berghella, V., ... \& Jacoby, V. L. (2020). Clinical presentation of coronavirus disease 2019 (COVID-19) in pregnant and recently pregnant people. Obstetrics and gynecology, 136(6), 1117.

Allotey, J., Stallings, E., Bonet, M., Yap, M., Chatterjee, S., Kew, T., ... \& PregCOV-19 Living Systematic Review Consortium. (2020). Clinical manifestations, risk factors, and maternal and perinatal outcomes of coronavirus disease 2019 in pregnancy: living systematic review and metaanalysis. Bmj, 370 .

Ashish, K. C., Gurung, R., Kinney, M. V., Sunny, A. K., Moinuddin, M., Basnet, O., ... \& Målqvist, M. (2020). Effect of the COVID-19 pandemic response on intrapartum care, stillbirth, and neonatal mortality outcomes in Nepal: a prospective observational study. The Lancet Global Health, 8(10), e1273-e1281.

Bradfield, Z., Hauck, Y., Homer, C. S., Sweet, L., Wilson, A. N., Szabo, R. A., ... \& Kuliukas, L. (2021). Midwives' experiences of providing maternity care during the COVID-19 pandemic in Australia. Women and Birth.

Brasil. Caderno de Atenção Básica: atenção ao pré-natal de baixo risco. Brasília, v.32, 2012. Disponível em: <https://www.google.com/url?sa=t\&rct= $\mathrm{j} \& \mathrm{q}=\& \mathrm{esrc}=\mathrm{s} \&$ source=web\&cd=\&ved=2ahUKEwjp8uWCqJDwAhW9rpUCHcn1CsQQFjAAegQIAhAD\&url=http\%3A\%2F\%2Fbvsms.saude.gov.br\%2Fbvs \%2Fpublicacoes\%2Fcadernos_atencao_basica_32_prenatal.pdf\&usg=AOvVaw290NvxUPOzIVj7RS8ycHHR>. Acesso em: 22 abr. 2021.

Cardim, M. Ministério da Saúde recomenda adiar gravidez devido a pico da Covid-19. Correio Braziliense, Distrito Federal, 16 de abr. de 2021. Disponível em: https://www.correiobraziliense.com.br/brasil/2021/04/4918657-ministerio-da-saude-recomenda-adiar-gravidez-devido-a-pico-da-covid-19.html.

Chen, D., Yang, H., Cao, Y., Cheng, W., Duan, T., Fan, C., ... \& Guan, X. (2020). Expert consensus for managing pregnant women and neonates born to mothers with suspected or confirmed novel coronavirus (COVID-19) infection. International Journal of gynecology \& obstetrics, 149(2), 130-136.

Chen, H., Guo, J., Wang, C., Luo, F., Yu, X., Zhang, W., ... \& Zhang, Y. (2020). Clinical characteristics and intrauterine vertical transmission potential of COVID-19 infection in nine pregnant women: a retrospective review of medical records. The lancet, 395(10226), 809-815.

Chen, Y., Peng, H., Wang, L., Zhao, Y., Zeng, L., Gao, H., \& Liu, Y. (2020). Infants born to mothers with a new coronavirus (COVID-19). Frontiers in pediatrics, 8,104 .

Chmielewska, B., Barratt, I., Townsend, R., Kalafat, E., van der Meulen, J., Gurol-Urganci, I., ... \& Khalil, A. (2021). Effects of the COVID-19 pandemic on maternal and perinatal outcomes: a systematic review and meta-analysis. The Lancet Global Health.

da Costa, R. E. A. R., Pompeu, J. G. F., Querido, Á. C. C. M., Campos, L. N. R., Calaça, M. B., da Silva, A. N., ... \& dos Santos Sousa, F. W. (2020). Principais Complicações Relacionadas à COVID-19 na Gravidez. Research, Society and Development, 9(8), e490985880-e490985880.

de Souza, H. C. C., de Matos, M. M. R., Costa, R. A., Lima, M. A. C., Cardoso, A. S., \& Bezerra, M. M. (2020). COVID-19 e gestação: manifestações clínicas, alterações laboratoriais e desfechos maternos, uma revisão sistemática de literatura. Brazilian Journal of Health Review, 3(6), 15901-15918.

Estrela, F., Silva, K. K. A. D., Cruz, M. A. D., \& Gomes, N. P. (2020). Gestantes no contexto da pandemia da Covid-19: reflexões e desafios. Physis: Revista de Saúde Coletiva, 30, e300215.

Gidlöf, S., Savchenko, J., Brune, T., \& Josefsson, H. (2020). COVID-19 in pregnancy with comorbidities: More liberal testing strategy is needed. Acta Obstet Gynecol Scand, 948-949.

Hoffmann, M., Kleine-Weber, H., Schroeder, S., Krüger, N., Herrler, T., Erichsen, S., ... \& Pöhlmann, S. (2020). SARS-CoV-2 cell entry depends on ACE2 and TMPRSS2 and is blocked by a clinically proven protease inhibitor. cell, 181(2), 271-280.

Meaney, S., Leitao, S., Olander, E. K., Pope, J., \& Matvienko-Sikar, K. (2021). The impact of COVID-19 on pregnant womens' experiences and perceptions of antenatal maternity care, social support, and stress-reduction strategies. Women and Birth.

Mendoza, M., Garcia-Ruiz, I., Maiz, N., Rodo, C., Garcia-Manau, P., Serrano, B., ... \& Suy, A. (2020). Pre-eclampsia-like syndrome induced by severe COVID-19: a prospective observational study. BJOG: An International Journal of Obstetrics \& Gynaecology, 127(11), 1374-1380.

Ministério da saúde. Secretaria de Vigilância em Saúde. Guia de Vigilância Epidemiológica: Emergência de Saúde Pública de importância nacional pela doença pelo Coronavírus 2019. Vigilância de Síndromes Respiratórias Agudas: COVID-19. Brasília - DF, 05 de ago. de 2020: 58p. Disponível em: <https://portalarquivos.saude.gov.br/images/af_gvs_coronavirus_6ago20_ajustes-finais-2.pdf〉. Acesso em: 22 abr. 2021.

Núbia, J. Sesau compra maternidade Regina Pacis por R $\$ 12$ milhões para atender infectados da COVID-19 em RO. G1, Rondônia, 07 de mai. de 2020. Disponível em: < https://g1.globo.com/ro/rondonia/noticia/2020/05/07/sesau-compra-maternidade-regina-pacis-por-r-12-milhoes-para-atender-infectados-dacovid-19-em-ro.ghtml>. Acesso em: 22 abr. 2021.

Oliviera, J. Ministério da Saúde recomenda que mulheres adiem a gravidez devido à pandemia. Pebmed, 2021. Disponível em: https://pebmed.com.br/ministerio-da-saude-recomenda-que-mulheres-adiem-a-gravidez-devido-a-pandemia/. Acesso em: 29 jul. 2021.

Peahl, A. F., \& Howell, J. (2020). The evolution of prenatal care delivery guidelines in the United States. American Journal of Obstetrics and Gynecology. 
Quansar, R., Dhkar, S. A., Saleem, S. M., \& Khan, S. M. S. (2020). Attitude and practices related to coronavirus disease (COVID-19) pandemic among pregnant women attending family welfare clinic amid Phase-2 lock down. Journal of Family Medicine and Primary Care, 9(12), 6085.

Rimmer, A. (2020). Covid-19: pregnant doctors should speak to occupational health, say experts. BMJ, $368, \mathrm{~m} 1104$.

Ruprecht, T. (2020). Hospitais de campanha: como vão funcionar e por que são tão importantes. Saúde. April, 9.

Sharps, M. C., Hayes, D. J., Lee, S., Zou, Z., Brady, C. A., Almoghrabi, Y., ... \& Heazell, A. E. (2020). A structured review of placental morphology and histopathological lesions associated with SARS-CoV-2 infection. Placenta, 101, 13-29.

Sheil, O., \& McAuliffe, F. M. (2021). Reorganisation of Obstetric services during the COVID pandemic-Experience from National Maternity Hospital Dublin Ireland. Best Practice \& Research Clinical Obstetrics \& Gynaecology.

Silva, A. W. C., Cunha, A. A., Alves, G. C., Corona, R. A., de Mattos Dias, C. A. G., Nassiri, R., ... \& Fecury, A. A. (2020). Caracterização clínica e epidemiologia de 1560 casos de COVID-19 em Macapá/AP, extremo norte do Brasil. Research, Society and Development, 9(8), e150985499-e150985499.

Sousa, G. O., Sales, B. N., Rodrigues, A. M. X., de Moura Rocha, G. M., \& de Oliveira, G. A. L. (2020). Evolução epidemiológica da COVID-19 no Brasil e no mundo. Research, Society and Development, 9(7), e630974653-e630974653.

Vasilevski, V., Sweet, L., Bradfield, Z., Wilson, A. N., Hauck, Y., Kuliukas, L., ... \& Wynter, K. (2021). Receiving maternity care during the COVID-19 pandemic: Experiences of women's partners and support persons. Women and Birth.

Vazquez-Vazquez, A., Dib, S., Rougeaux, E., Wells, J. C., \& Fewtrell, M. S. (2021). The impact of the Covid-19 lockdown on the experiences and feeding practices of new mothers in the UK: Preliminary data from the COVID-19 New Mum Study. Appetite, 156, 104985.

Wang, C. L. (2021). Impact of COVID-19 on Pregnancy. International Journal of Medical Sciences, $18(3), 763$.

Zhang, L., Dong, L., Ming, L., Wei, M., Li, J., Hu, R., \& Yang, J. (2020). Severe acute respiratory syndrome coronavirus 2 (SARS-CoV-2) infection during late pregnancy: a report of 18 patients from Wuhan, China. BMC Pregnancy and Childbirth, 20(1), 1-7 